\title{
Hipec in Ovarian Cancer. Why is it Still the Ugly Duckling of Intraperitoneal Therapy?
}

Pedro Antonio Cascales Campos*, Jose Gil, Pablo Munoz-Ramon and Pascual Parrilla

Hospital Universitario Virgen de la Arrixaca, Department of Surgery, Murcia, Spain

\section{Dear Editor,}

What kind of intraperitoneal therapy would be recommend for our mother, sister, wife or daughter if she was diagnosed with advanced ovarian cancer and if we were in the office of an experienced surgeon able to achieve an optimal resection or, even better, a complete resection of her disease?

If one was a surgeon who believed in postoperative systemic chemotherapy, one's argument would be based on the results of the study GOG 172 (which coincided with a clinical alert from the NCI of the United States in favor of this modality of intraperitoneal treatment after optimal surgery in patients with ovarian cancer) [1]. According to the paper published by Armstrong et al, our relative would have a median global survival of more than 65.6 months. However, the possibilities of successfully accomplishing the protocol would be very low $(42 \%)$ as a result of severe systemic complications related to the treatment, even if there were no problems with the infusion catheter. In addition, several modified protocols have been reported in order to reduce problems as those seen in the GOG 172 study but actually IP/ IV chemotherapy use in clinical practice is an underused strategy to improve ovarian cancer outcomes.

On the contrary, if one was a surgeon who believed in hypertermic intraperitoneal chemotherapy (HIPEC) and one was asked the scientific basis on which its use in ovarian cancer is based, this surgeon would say that, even if there are no prospective and randomized studies which recommend its widespread use, the risks of morbidity and mortality are perfectly acceptable and that, in addition, some studies have demonstrated the utility of HIPEC for the treatment of residual disease after the surgery, with one phase III and several phase II trials which demonstrate five-year survival rates higher that $60 \%$ [2-4].

Once we had taken into account both opinions, our relative would still have a doubt about which one is better for her. Each surgeon will have defended his or her opinion exposing the weak points of the other option. The first surgeon will have a stagnant attitude and so he or she will focus on the lack of scientific evidence (which by the way, will be coming soon). However, the necessary undeniable scientific evidence is sometimes a double-edged sword. No prospective randomized clinical trials in favor of resection of hepatic metastases secondary to CCR exist either. The use of Bevacizumab in combination with platinum and taxanes as a first line treatment for advanced ovarian cancer is just justified with "statistical engineering" [5]. Afilibercept + FOLFIRI in patients with colorectal cancer in stage IV has demonstrated an increase in global survival of only 44 days [6]. Only time will put HIPEC in the place it belongs to. Meanwhile, give us the opportunity of turning HIPEC into a gorgeous swan.

\section{References}

1. Armstrong DK, Bundy B, Wenzel L, et al. (2006) Intraperitoneal cisplatin and paclitaxel in ovarian cancer. N Engl J Med 354: 34-43.

2. Cascales-Campos PA, Gil J, Gil E, Feliciangeli E, Gonzalez-Gil A, et al. (2014) Treatment of microscopic disease with hyperthermic intraoperative intraperitoneal chemotherapy after complete cytoreduction improves diseasefree survival in patients with stage IIIC/IV ovarian cancer. Ann Surg Oncol 21: 2383-2389.

3. Deraco M, Kusamura S, Virzi S, Puccio F, Macri A, et al. (2011) Cytoreductive surgery and hyperthermic intraperitoneal chemotherapy as upfront therapy for advanced epithelial ovarian cancer: multi-institutional phase-II trial. Gynecol Oncol 122: 215-220.

4. Shu CA, Konner JA (2015) Breaking Down the Evidence for Bevacizumab in Ovarian Cancer. Oncologist 20: 91-93.

5. Spiliotis J, Halkia E, Lianos E, Kalantzi N, Grivas A, et al. (2015) Cytoreductive surgery and HIPEC in recurrent epithelial ovarian cancer: a prospective randomized phase III study. Ann Surg Oncol 22: 1570-1575.

6. Van Cutsem E, Tabernero J, Lakomy R, Prenen H, Prausova J, et al. (2012) Addition of aflibercept to fluorouracil, leucovorin, and irinotecan improves survival in a phase III randomized trial in patients with metastatic colorectal cancer previously treated with an oxaliplatin-based regimen. J Clin Oncol 30: 3499-3506.
*Corresponding author: Cascales PAC, Hospital Universitario Virgen de la Arrixaca, Department of Surgery, Ctra. Madrid-Cartagena, s/n, 30120 El Palmar Murcia, Spain, Tel: +34968369500; E-mail: cascalescirugia@gmail.com

Received December 21, 2015; Accepted February 10, 2016; Published February 12, 2016

Citation: Cascales Campos PA, Gil J, Munoz-Ramon P, Parrilla P (2016) Hipec in Ovarian Cancer. Why is it Still the Ugly Duckling of Intraperitoneal Therapy? J Cancer Sci Ther 8: 030. doi:10.4172/1948-5956.1000383

Copyright: (C) 2016 Cascales Campos PA, et al. This is an open-access article distributed under the terms of the Creative Commons Attribution License, which permits unrestricted use, distribution, and reproduction in any medium, provided the original author and source are credited. 